\title{
coviD-19 Towards a standardised method of patient prioritisation that accounts for clinical harm
}

\author{
Authors: Rowan Wathes, ${ }^{\mathrm{A}}$ Karina Malhotra, ${ }^{\mathrm{B}}$ Kelsey Flott, ${ }^{\mathrm{C}}$ Arup Nath $^{\mathrm{A}}$ and Catherine Urch ${ }^{\mathrm{D}}$
}

\begin{abstract}
COVID-19 has highlighted the need for a standardised approach for prioritising patients requiring elective care. The Royal College of Surgeons of England (RCS) developed guidance at the start of the pandemic for prioritising surgical patients based on the urgency of different procedures. Imperial College Healthcare NHS Trust (ICHT) has extended this to all aspects of elective care to enable standardised decision-making based on clinical priority, clinical harm and patient vulnerability. This was a clinically led project that involved close collaboration with lay partners, who were concerned that the RCS guidance lacked the sensitivity to reflect individual patients' needs. Our novel elective care recovery matrix is designed to be applicable across all elective care services and at Trust or system level. Implementation at ICHT progressed rapidly: as of 28 August $\mathbf{2 0 2 0}>\mathbf{2 0 0}$ consultants have received training on the process and $58 \%$ of all surgical orders have been prioritised using the new framework $(5,134 / 8,800)$. While COVID-19 was the driver, the applicability can be wider and could inform new ways of working. The framework enables rapid quantification of individual patient care requirements, thus enabling clinicians to target more accurately those patients with the greatest need and those who would see the greatest benefit.
\end{abstract}

KEYWORDS: COVID-19, prioritisation, elective care, cancer, framework

DOI: 10.7861/fhj.2020-0109

\section{Problem}

It is widely accepted that COVID-19 will be endemic in the UK for at least 18-24 months and will continue to consume significant health and social care capacity and resources. ${ }^{1}$ As we move from a pandemic emergency response towards a new normal, we need a way of prioritising the huge backlog of patients requiring elective care. The number of referral to treatment (RTT) patients waiting to

Authors: ${ }^{A}$ consultant, The PSC, London, UK; ${ }^{B}$ RTT improvement partner, Acumentice Health, London, UK; Cpolicy fellow and patient safety lead, Imperial College London, London, UK; 'D divisional director for surgery, cardiovascular and cancer and trust cancer lead, Imperial College Healthcare NHS Trust, London, UK start treatment at the end of June 2020 was 3.9 million patients and it is estimated that waiting lists could increase by more than $100 \%$ to over 9 million over the next 12 months. ${ }^{2,3}$ Of those on the waiting list in June, 50,536 patients had been waiting more than 52 weeks.

During COVID-19 every effort has been made to review the surgical 'to be scheduled' lists, and outpatient appointment lists have been reviewed to identify those patients who need emergency treatment. However, trusts often have limited records of these reviews, which are often done on spreadsheets, and even less understanding of how some patients are re-prioritised.

Traditional methods for prioritising elective care in the UK are unidimensional, based on time waited, eg RTT or the 62-day cancer standard. They rely on retrospective reviews once the patient has already waited or breached a performance target, and do not account for future projected waiting. They can drive a preoccupation with targets rather than clinical need. Furthermore, they do not allow for the re-prioritisation of patients with the same diagnostic code but different clinical requirements.

The patient safety evidence base suggests that this is not the safest approach to prioritisation and can result in inadvertent harm to patients or clinical deterioration. In the UK, studies have started to demonstrate the value of more patient-centric approaches to prioritisation of elective procedures by using data such as Patient Reported Outcome Measures (PROMs). ${ }^{4}$ Examples from health systems internationally demonstrate the benefit of more dynamic approaches to clinical risk prioritisation in a range of settings. For instance, studies from New Zealand provide evidence of how risk prioritisation can take account of a more diverse dataset, including quality-of-life information. ${ }^{5}$ Studies from Italy go further to suggest the need to engage with waitlists in a fluid and responsive way to prioritise and re-prioritise elective patients. ${ }^{6}$ Despite the growing evidence on this topic, a recent systematic review reveals a lack of conclusive evidence about best practice in clinical risk prioritisation for elective patients, providing part of the impetus for this study. ${ }^{7}$

\section{Potential solution}

The Royal College of Surgeons of England (RCS), the British Society of Gastroenterology and the Joint Advisory Group produced guidance on the prioritisation of patients for surgery and endoscopy respectively at the start of the pandemic..$^{8,9}$ The principle underpinning their guidance is a time-based assessment of the urgency of provision. Imperial College Healthcare NHS Trust (ICHT) has developed a novel framework that extends this principle to all aspects of elective clinical care and enables clinicians to take actual or potential clinical harm into account. 


\begin{tabular}{|c|c|c|c|c|c|}
\hline Level of clinical & Clinical priority & & & & \\
\hline & $\begin{array}{l}\text { Priority } 1 \mathrm{a}:<24 \mathrm{hrs} \\
\text { Priority } 1 \mathrm{~b}:<72 \mathrm{hrs}\end{array}$ & $\begin{array}{l}\text { Priority } 2:<1 \\
\text { month (urgent and } \\
\text { cancer) }\end{array}$ & $\begin{array}{l}\text { Priority } 3:<3 \\
\text { months (routine } \\
\text { expedited) }\end{array}$ & $\begin{array}{l}\text { Priority } 4:>3 \\
\text { months (routine) }\end{array}$ & $\begin{array}{l}\text { Adapt or bespoke investigation/ } \\
\text { treatment site/follow up }\end{array}$ \\
\hline None & $\mathrm{n} / \mathrm{a}$ & $\begin{array}{l}\text { Stay P2 } \\
\text { PTL review by } 3 \\
\text { months }\end{array}$ & $\begin{array}{l}\text { Stay P3 } \\
\text { PTL review by } 6 \\
\text { months }\end{array}$ & $\begin{array}{l}\text { Stay P4 } \\
\text { PTL review by } 12 \\
\text { months }\end{array}$ & $\begin{array}{l}\text { Adapt or bespoke investigation/ } \\
\text { treatment site/follow up }\end{array}$ \\
\hline Mild & $\mathrm{n} / \mathrm{a}$ & $\begin{array}{l}\text { Stay P2 } \\
\text { PTL review by } 3 \\
\text { months }\end{array}$ & $\begin{array}{l}\text { Stay P3 } \\
\text { PTL review by } 6 \\
\text { months }\end{array}$ & $\begin{array}{l}\text { Stay P4 } \\
\text { PTL review by } 12 \\
\text { months }\end{array}$ & $\begin{array}{l}\text { Adapt or bespoke investigation/ } \\
\text { treatment site/follow up }\end{array}$ \\
\hline Moderate & $\begin{array}{l}\text { Stay P1 } \\
\text { PTL review daily or } \\
\text { weekly }\end{array}$ & $\begin{array}{l}\text { Stay P2 } \\
\text { PTL review by } 1 \\
\text { month }\end{array}$ & $\begin{array}{l}\text { NEW P2 } \\
\text { PTL review by } 1 \\
\text { month }\end{array}$ & $\begin{array}{l}\text { NEW P3 } \\
\text { PTL review by } 3 \\
\text { months }\end{array}$ & $\begin{array}{l}\text { Adapt or bespoke investigation/ } \\
\text { treatment site/follow up }\end{array}$ \\
\hline Severe & $\begin{array}{l}\text { NEW P1a PTL } \\
\text { Review daily }\end{array}$ & $\begin{array}{l}\text { NEW P1 b } \\
\text { PTL review daily or } \\
\text { weekly }\end{array}$ & $\begin{array}{l}\text { NEW } \mathrm{P} 2 \text { or } \mathrm{P} 1 \mathrm{~b} \\
\text { PTL review by } 1 \\
\text { month }\end{array}$ & $\begin{array}{l}\text { NEW } \mathrm{P} 2 \text { or } 1 \mathrm{~b} \\
\text { PTL review by } 1 \\
\text { month }\end{array}$ & $\begin{array}{l}\text { Adapt or bespoke investigation/ } \\
\text { treatment site/follow up }\end{array}$ \\
\hline
\end{tabular}

Fig 1. The standardised matrix, combining priority and clinical harm. PTL = patient tracking list.

Following a series of discussions with clinical colleagues and lay partners, who were concerned that the NHSE guidance lacked the sensitivity to reflect individual patients' needs, a prototype matrix was developed and tested with further stakeholders and at the ICHT Clinical Reference Group. This testing resulted in further iteration of the matrix and its application, and the development of standardised harm definitions. While development of the matrix was driven by the COVID-19 pandemic, it was developed using evidence-based clinical priorities and is applicable at any time of constrained resources and in any elective care setting.

The matrix (Fig 1), combined with standardised clinical priority categories (Priority 1-4, or P1-4) and standardised definitions of clinical harm (Table 1), provides a resource to guide decision making and support the generation of 'outcome priorities', with corresponding actions. The matrix enables rapid quantification of individual patient care requirements, thus enabling clinicians

Table 1. Summary of clinical harm definitions and how each clinical harm definition can be used to modify the priority category of each patient

\section{Clinical \\ Definition}

harm rating

None

Neither current wait nor proposed deferral of investigation or treatment will cause organ damage or alter management

Mild

No actual harm caused by current wait but proposed deferral may cause limited harm (no organ damage or change in prognosis but may impact on psychological wellbeing or functional status)

Moderate Current wait has caused mild actual harm or

Proposed deferral may cause moderate harm in terms of organ damage, altered prognosis, change in treatment options, reduced functional status, severe pain and/or significant psychological distress

Severe Current wait has caused moderate actual harm or

Proposed deferral may cause severe harm in terms of organ damage, altered prognosis, change in treatment options, reduced functional status, severe pain, overwhelming psychological distress, and/or treatment intent changed to palliative/terminal care only

\section{Recommended action(s)}

Consider discharging to primary care with appropriate safety netting. If not appropriate, continue with existing Priority category and review pathway annually

Consider discharging to primary care with appropriate safety netting. If not appropriate, continue with current Priority category and schedule next event (accounting for time already waited)

Move up a Priority category (from current category) and schedule next event (accounting for time already waited) Alert patient and GP

Move up a Priority category (from current category) and consider if harm warrants escalation to $\mathrm{P} 1 \mathrm{~b}$

Alert patient and GP

Ensure active tracking at least weekly

RTT $=$ Referral to Treatment 
Box 1. Examples of how the matrix can be used operationally

Note: A patient with uncomplicated renal stones would usually be categorised as P3 for surgery (ie the patient can be deferred for up to 3 months).

Example 1: The patient above, who has already been deferred for 20 weeks, now has mildly impaired renal function (obstructive uropathy). The wait has induced mild actual harm, and with further waiting at P3, there is a high risk of moderate actual harm. Using the matrix, we navigate to the P3/moderate harm box and can see that the recommended action is to recategorise this patient as P2 (the 'outcome priority' is P2). This drives the expedition of any investigations and interventions from within 3 months to within 1 month.

Example 2: The patient above presents with severely impaired renal function (severe actual harm). Using the matrix, we navigate to the P3/severe harm box and can see that the recommended action is to re-categorise the patient as $\mathrm{P} 2$ or $\mathrm{P} 1 \mathrm{~b}$ (the decision between $\mathrm{P} 2$ and $\mathrm{P} 1 \mathrm{~b}$ would be a clinical decision). This drives the expedition of any investigations and interventions from within 3 months to within 1 month (P2) or within 72 hours (P1b).

Example 3: There is an incidental finding of renal stones in a patient with complete renal failure who is on haemodialysis. The patient has no pain or infection. There has been no actual harm and the clinical assessment is that deferral would be unlikely to cause harm (eg due to pain or infection). This patient might be categorised as $\mathrm{P} 4$ as the management of renal stones is not required to save renal function. The patient could potentially be discharged to primary care with appropriate safety-netting for infection risk or pain.

to target more accurately those patients with the greatest need and those who would see the greatest benefit. It can be used at the level of the trust or system to generate patient tracking lists (PTLs).

Once generated, the outcome priorities and actions can be adjusted according to COVID-19 vulnerability and additional patient needs. This modification was added following consultation with patients and lay partners. The presence or absence of vulnerability does not alter the clinical priority, nor the level of clinical harm. However, it will alter the balance between the risk of treatment during the pandemic and the risk of deferral, the recommended management options, the place of treatment, the consent process and the scheduling of follow-up. Ultimately, the decision about whether to proceed or defer will need to be made by the clinician responsible for the patient and the patient themselves. Patients must be appropriately consented, and any discussions or decisions must be clearly documented. See Box 1 for examples of how the matrix can be used operationally.

The framework redefines waiting times, not according to a time waited per se (RTT), but rather by clinical harm (actual or potential). However, the length of time an individual has already waited will be important in reviewing harm. In addition, as capacity or resource is expanded, the length of waiting will become an important feature in prioritising $\mathrm{P} 3$ and $\mathrm{P} 4$ patients. The framework will also enable a significant number of patients waiting for routine procedures or investigations that will not alter prognosis, organ function or functional status to be reprioritised within a priority category, move priority category and/ or potentially be discharged to primary care with appropriate safety-netting.

Operationalising this approach may seem daunting. For example, at ICHT the RTT Patient Tracking List comprises almost 60,000 pathways and there are estimated to be several hundred thousand non-RTT pathways. However, implementation at ICHT is progressing rapidly due to the robust and innovative implementation plan, centred around four key elements (Box 2). The matrix is being used to embed a culture change in how all patients waiting for investigations, outpatient appointments or treatment are managed. It is being rolled out in phases, starting with surgical orders (Phase 1). Phase 2 will cover all diagnostic orders and Phase 3 will cover all other outpatient activity. The framework builds on the RCS clinical priorities, with effective safety mechanisms so that patients are not 'lost' within scheduling processes and are re-prioritised as required based on individual factors such as harm or vulnerability to COVID-19.

\section{Results so far}

Training commenced on 29 June 2020 and as of 28 August 2020, when this paper was written, over 200 consultants have received one-to-one training sessions through a virtual collaboration platform. This represents coverage of approximately $89 \%$ within Surgery (using named clinicians as a proportion of patients on the surgical RTT inpatient waiting list as a proxy for coverage). Training is quick as the process is so intuitive: on average, each technical application session lasts 12 minutes, and most of that time is spent setting up clinical worklists on Cerner, the electronic patient record system.

In July, it became mandatory to add a clinical priority to all Cerner surgical orders and as of 28 August $2020,58 \%$ of all surgical orders had been prioritised using the new framework $(5,134 / 8,800)$. This will quickly increase to $100 \%$ as the backlog is cleared. The priorities are already enabling better tracking and safety-netting of patients. For example, all patients listed as 'P2' are regularly cross-checked against 'to come in' (TCI) Lists to ensure their surgery is scheduled to occur within a month, and a clinical harm review is triggered if the surgery is not scheduled to occur within 8 weeks of prioritisation. If harm is recorded and a patient is reprioritised because of this, this triggers a Duty of Candour conversation between the clinician and the patient. To date, no Duty of Candour conversations have been triggered. A report summarising the Trust's position is automatically generated and sent to the relevant managerial staff on a weekly basis. It is also discussed at the Elective Surgery Recovery Group, the Elective Care Recovery Board and the Trust's Quality and Safety Group.

To ensure that no patients were being deprioritised inappropriately due to implementation of the framework, an audit was conducted. A sample of over 100 patients was randomly selected, and their notes and clinic letters were reviewed by a senior consultant within the specialist area. 100\% P3 and P4, and $>90 \% \mathrm{P} 2$, were correctly categorised (the difference was in the local consultant's understanding of the harm rating and therefore movement of patients). The audit has triggered updates to the training materials and targeted sessions with the relevant consultants.

An interesting by-product of the work has been improvements to the data quality of waiting lists. Surgeons are reviewing their full surgical waiting lists (which has not always been the case), allowing them to feed back rapidly if patients have already received treatment (especially at another trust) but are still 
Box 2. Approach to implementation

\section{IT changes}

Changes were made to the Trust's patient administration system (PAS) to enable clinical priorities to be recorded and linked to the electronic patient record system (Cerner). Previously, clinical priorities were held in spreadsheets, which add minimal value. A mandatory field for prioritisation was added to the surgical order form. This meant that prioritisation was built into the clinical workflow. It made it impossible to schedule an operation or procedure without documenting the clinical priority. It enabled the aggregation of clinical prioritisation data at different levels (eg department, division). This data has been an invaluable input into recovery planning and decision making around use of capacity and procurement of additional capacity.

\section{Training}

Training focused on both the clinical use of the matrix and technical aspects. Regarding clinical use, large classroom sessions were arranged, during which the project's clinical lead talked through the purpose and use of the matrix, using clinical cases to highlight key aspects. These were recorded so clinicians could watch them in their own time. Regarding the technical aspects, training materials were developed and eight student volunteers were trained to facilitate one-to-one remote session with the consultants, which were mainly focused on the process of setting up clinical worklists in Cerner.

\section{Embedding the change in policies and procedures}

The process for scheduling was iterated: schedulers were asked to schedule based on the clinical priorities on Cerner. Consultants were told that the only way to expedite a patient's treatment was through changing the clinical priority on Cerner. This meant changes to the lists were fair and transparent. The process was also linked to the process for reviewing clinical harm. For example, all patients listed as 'P2' are regularly cross-checked against 'to come in' (TCI) lists to ensure their surgery is scheduled to occur within a month, and a clinical harm review is triggered if the surgery is not scheduled to occur within 8 weeks of prioritisation.

\section{Governance}

Robust governance was established to support safe and rapid implementation of the new process. A clinical prioritisation working group was set up to plan and monitor implementation. Key performance metrics (such as number of P2s without a TCI) are presented at the existing Elective Surgery Recovery Group to support targeted problem solving. Key performance metrics, and any knotty issues, are discussed at the Elective Care Recovery Board and ultimately the Trust's Quality and Safety Group.

appearing as 'waiting for treatment'. This is making it easier for schedulers to schedule dates for patients who are genuinely waiting. We estimate that approximately $5-10 \%$ of patients on the surgical inpatient waiting list have been appropriately removed as a result of this process. There are also anecdotal reports that the individual assessments of need through use of the framework are helping to address health inequalities. This will require further research and quantification.

The methodology is spreading across North West London and training has commenced at Chelsea and Westminster Hospital NHS Foundation Trust. The aim is to increase coverage further to enable system-wide prioritisation and the development of a system-wide PTL.

\section{Conclusion and potential future}

The concept of using clinical harm to prioritise patients is not new and is familiar to most clinicians - however, the framework, the standardised definitions, and their wide application to drive equity of care are new. The concept and the definitions herein have been discussed with a wide variety of healthcare colleagues, including radiologists, surgeons, oncologists and others working at ICHT and within the local system. There is broad agreement around their value and commitment within ICHT and North West London to make further improvements to the way patients are prioritised. Future iterations of the framework could take account of a broader definition of need and perhaps set the discussion in the context of a need for greater overall efficiency and productivity and understanding of true demand/responsiveness of elective care to patient need and preferences.

As it currently stands, the framework goes a long way towards enabling the equitable prioritisation of patients in different settings, with different diagnoses, and even under the care of different trusts. It enables a logical and consistent approach to help clinicians and managers alike to prioritise and manage their patients. It is flexible and relies on clinical judgement, but the standardised definitions enable external scrutiny and moderation within and between teams, and across health systems.

The framework enables a system-wide PTL, with outcome actions, to be generated to focus limited resources to the greatest effect. It is designed to be used during COVID-19, but it could form the basis for prioritising all patients for intervention or review in a resource constrained environment, regardless of the presence of COVID-19 or any other pandemic.

\section{Acknowledgements}

The authors thank the NIHR Patient Safety Translational Research Centre at Imperial College London.

\section{References}

1 Moore KA, Lipsitch M, Barry JM, Osterholm MT. COVID-19: The CIDRAP Viewpoint. Part 1: The future of the COVID-19 pandemic: lessons learned from pandemic influenza. 2020. Center For Infectious Disease Research and Policy, 2020. www.cidrap.umn. edu/sites/default/files/public/downloads/cidrap-covid19-viewpointpart1_0.pdf.

2 NHS England. Statistical Press Notice NHS referral to treatment (RTT) waiting times data June 2020. NHSE, 2020. www.england.nhs. uk/statistics/wp-content/uploads/sites/2/2020/08/RTT-statisticalpress-notice-PDF-357K-24258.pdf [Accessed 17 September 2020].

3 Wood R. Modelling the impact of COVID-19 on elective waiting times. J Simulation 2020, in press (doi: 10.1080/17477778.2020.1764876).

4 Chalkley M, McCormick B, Anderson R et al. Prioritising patients for elective surgery: the efficiency of alternative selection criteria. In: 
Elective hospital admissions: secondary data analysis and modelling with an emphasis on policies to moderate growth (Health Services and Delivery Research 5.7). NIHR, 2017.

5 Derrett S, Devlin N, Hansen P et al. Prioritizing patients for elective surgery: a prospective study of clinical priority assessment criteria in New Zealand. Int J Technol Assess Health Care 2003;19:91-105.

6 Valente R, Testi A, Tanfani E et al. A model to prioritize access to elective surgery on the basis of clinical urgency and waiting time. BMC Health Serv Res 2009;9:1.

7 MacCormick A, Collecutt W, Parry B. Prioritizing patients for elective surgery: a systematic review. ANZ J Surg 2003;73:633-42.

8 Federation of Surgical Specialty Associations. Clinical guide to surgical prioritisation during the coronavirus pandemic. FSSA, 2020. https://fssa.org.uk/_userfiles/pages/files/covid19/prioritisation_ master_240720.pdf.

9 British Society of Gastroentorology. Endoscopy activity and COVID19: BSG and JAG guidance. BSG, 2020. www.bsg.org.uk/covid-19advice/endoscopy-activity-and-covid-19-bsg-and-jag-guidance.

Address for correspondence: Dr Rowan Wathes, Cancer Services, St Mary's Hospital, Imperial College Healthcare NHS Trust, Praed Street, Paddington, London W2 1NY, UK. Email: rowan.wathes@gmail.com 